\title{
Dense, Strongly-Interacting Matter: Strangeness in Heavy-Ion Reactions 1-10 A GeV
}

\author{
C.A. Ogilvie \\ Massachusetts Institute of Technology, Cambridge, MA 02139
}

\begin{abstract}
This talk reviews the physics of dense, strongly interacting matter. The newly acquired experimental data on strangeness production in heavy-ion reactions from 1-10 AGeV is used to probe the physics of in-medium properties of matter, multi-body collisions, and the search for the baryon-rich quark-gluon plasma.
\end{abstract}

\section{INTRODUCTION}

In 1996 the heavy-ion community explored a whole new physics landscape by measuring $\mathrm{Au}+\mathrm{Au}$ reactions at beam energies of 2, 4, 6 and $8 \mathrm{AGeV}$. With experimental data to link the AGS full-energy of $10 \mathrm{AGeV}$ and the BEVALAC/SIS energies of 1-2 AGeV, we can uniquely explore how the physics of strongly-interacting matter changes as a function of density. The results from experiments E895 and E866/E917 @ AGS and KaoS @ SIS are becoming available, so it is timely to review the physics of strange particle production across the energy range from SIS to AGS.

There are at least three interconnected physics questions about how matter of increasing density behaves; 1) what are the appropriate degrees-of-freedom?, multiply-colliding hadrons and their excited resonances, or is the matter dense enough such that a baryon-rich quarkgluon plasma [1] is formed, 2) if the system remains in the hadronic sector, do the hadrons propagate with an effective mass different than their free mass [2], and 3) do multi-body, 
instead of two-body, hadronic collisions play a role in the reaction dynamics [3]?

Before reviewing the data and the status of answers to these physics questions, I would like to expand on each of the three topics.

In contrast to the high-temperature QGP where quarks and gluons are predicted to be quasi-free, the baryon(quantum-number)-rich plasma is conjectured to have momentumspace correlations between pairs of quarks 4 . This pairing is predicted to produce a colorsuperconducting quark-gluon phase of matter. The transitions between this phase, the quasi-free quark-gluon phase, and normal hadronic matter could make a rich and varied phase diagram of strongly-interacting matter. However there is currently little knowledge of what signatures could indicate the onset of the superconducting quark phase, or whether the standard battery of high-temperature QGP signatures would retain their sensitivity in the baryon-rich phase.

Dense matter, which at several times normal nuclear density $\left(\rho \sim 0.6-0.7 \mathrm{fm}^{-3}\right)$ may be insufficient to form a quark-gluon plasma, should still be considered a novel and fascinating system in its own right. At four times normal density the mean-free path $(\lambda=1 / \rho \sigma)$ of typical hadrons is a strikingly small $0.5 \mathrm{fm}$. This estimate of the mean free path is smaller than the typical size of a hadron which implies that the system is so densely packed that each hadron can collide several times per $1 \mathrm{fm} / \mathrm{c}$. Such multiple collisions will not be independent of each other and could behave differently than a succession of two-body collisions [3].

At considerably lower densities there are several predictions [5] that kaons have inmedium properties. $\mathrm{K}^{-}$are predicted to interact attractively with baryons, which can be implicitly accounted for by either an attractive mean-field [6] or by decreasing the $\mathrm{K}^{-}$effective mass with density [5]. $\mathrm{K}^{+}$are expected to have a repulsive interaction with baryons [6.77. In transport calculations of heavy-ion reactions, these in-medium effects are predicted to strongly increase the yield of $\mathrm{K}^{-}$due to the increase of phase-space from the drop in $\mathrm{K}^{-}$mass. The spectra of $\mathrm{K}^{+}$and $\mathrm{K}^{-}$are also predicted to be shifted in opposite directions [8.6], with $\mathrm{K}^{+}$repelled to higher transverse momentum and $\mathrm{K}^{-}$attracted to lower values of transverse momentum. 
By changing the density it may be possible, but very difficult, to identify any densitydependent in-medium effect and separate this from other processes that affect kaon production, e.g. multiple secondary collisions. To establish the existence of in-medium physics will require the systematic use of the measured $\mathrm{K}^{+}$and $\mathrm{K}^{-}$yields and spectra as a function of beam-energy. As a strong consistency check, any posited explanation of an experimental result that supports in-medium physics, must also explain data at other beam energies, as well as the other observables that are sensitive to in-medium effects.

By studying strangeness production as a function of beam energy we can probe these three interlinked physics questions: in-medium properties, multi-body collisions, and the baryon-rich QGP. This will help us make progress towards a consistent understanding of the behavior of strongly-interacting matter as a function of density.

\section{STRANGE YIELDS IN REACTIONS 1-10 A GEV}

In Figure 1 the yield of $\mathrm{K}^{+}$per projectile participant is plotted versus the number of projectile participants in $\mathrm{Au}+\mathrm{Au}$ reactions at the full AGS energy of 11.6AGeV [9]. The number of participants is calculated from the measured energy of the projectile spectators. The yield per projectile participant in central reactions is 3.5 times larger than the $\mathrm{K}^{+}$yields from the isospin-averaged N-N collisions at the same beam energy. This extra production steadily increases with the number of projectile participants and hence most probably comes from multiple secondary collisions of hadrons in a heavy-ion reaction 10 12. This mechanism is aided by many of the secondary collisions being between one or two hadronic resonances with the energy of the resonances available for kaon production.

In $\mathrm{Au}+\mathrm{Au}$ collisions at $1 \mathrm{AGeV}$ [13], $\mathrm{K}^{+}$production per participant also increases with centrality (Figure 2), suggesting that a similar secondary collision production mechanism may work at both SIS and AGS energies. The pion yield per participant (Figure 2) is nearly independent of centrality. It is possible that the difference between pions and kaons is the strong absorption of pions. 
The kaon yield can be parameterized as a power law in the number of participants

$$
\text { yield }=\mathrm{a} \times \mathrm{N}_{\mathrm{pp}}^{\alpha}
$$

At SPS energies, where the centrality dependence of kaons yields is not yet available, the power-law exponent for $\Lambda$ production is used instead [14]. The power-law exponent $\alpha$ decreases with higher beam energies (Figure 3). If kaon enhancement is defined as the yield in central collisions compared to either peripheral collisions, or N-N reactions, then the enhancement of strangeness is largest at the lower beam energies. This is consistent with secondary collisions increasing in relative importance for kaon production compared to initial collisions as the beam energy is reduced.

The change in the power-law exponent for $\mathrm{K}^{+}$production demonstrates that heavy-ion yields have a different beam energy dependence than proton-proton reactions. Secondary collisions will also change the energy dependence of the $\mathrm{K}^{-}$yield. This is an effect which will need to be firmly understood before any in-medium mass reduction of $\mathrm{K}^{-}$can be inferred from the yield of $\mathrm{K}^{-}$or the shape of its excitation function [15].

It is instructive to compare $\mathrm{K}^{-}$and $\mathrm{K}^{+}$production by plotting the ratio of total yields as a function of the number of participants. Figure 4 shows the $\mathrm{K}^{-} / \mathrm{K}^{+}$ratio in $\mathrm{Au}+\mathrm{Au}$ reactions at $11.6 \mathrm{AGeV} / \mathrm{c}$ [9], and figure 5 plots this ratio for $\mathrm{Ni}+\mathrm{Ni}$ reactions at $1.8 \mathrm{AGeV}$ [15]. At both beam energies, and also at the intermediate energy of $4 \mathrm{AGeV}$, the $\mathrm{K}^{-} / \mathrm{K}^{+}$ ratio is independent of centrality. Whatever mechanism drives the enhancement of kaons with centrality, it similarly affects the production of both $\mathrm{K}^{+}$and $\mathrm{K}^{-}$.

Given the many differences between $\mathrm{K}^{-}$and $\mathrm{K}^{+}$production, absorption, and possible opposite changes of in-medium mass, the observation that the $\mathrm{K}^{-} / \mathrm{K}^{+}$ratio is constant with centrality is a puzzle. At each beam energy, the volume of dense matter, and even the density should increase with centrality. Any possible $\mathrm{K}^{-}$mass decrease with density should lead to an increase in the $\mathrm{K}^{-} / \mathrm{K}^{+}$ratio with centrality. This is not observed, potentially placing a strong constraint on the existence of in-medium kaon properties.

A review of the theoretical effort to model these reactions is outside the scope of this 
paper. For our purposes we can divide the models into two classes, those that include the possibility of hadrons having an effective in-medium mass (HSD [7], RBUU [16], ART [12]) and those that use normal masses throughout (RQMD [10], UrQMD [17] and the above codes run in normal-mass mode).

Calculations that use in-medium masses successfully reproduce the experimental yield of $\mathrm{K}^{-}$to within $30 \%$ at both GSI [16,18] and AGS [18] energies. The $\mathrm{K}^{-} / \mathrm{K}^{+}$ratio has also been studied [16]. The calculated ratio increases with centrality when in-medium masses are used, however the predicted effect is negated by the increased absorption of $\mathrm{K}^{-}$in dense matter. While such a cancelation is plausible, it would be unusual for the cancelation to occur at all energies from 1 to $10 \mathrm{AGeV}$ and as such, the explanation should be checked across this full energy range.

No consistent picture has yet to emerge on the calculated $\mathrm{K}^{+}$yields. Using an increasing in-medium mass for $\mathrm{K}^{+}$, transport calculations underpredict the measured yield by factors of two [18]. As a cross-check on the consistency of $\mathrm{K}^{+}$production in these models, RQMD, HSD, and ART calculations with unmodified, normal masses for $\mathrm{Au}+\mathrm{Au}$ reactions at 11.6 $\mathrm{AGeV} / \mathrm{c}$ are compared in figure 6. RQMD impressively reproduces the centrality dependence of kaon production, whereas both ART and HSD, for which only central calculations are available, produce too few kaons. These calculations nominally include the same physics, i.e. multiple scattering and excitation of a range of resonances, yet for central collisions there is a factor of two discrepancy between the predictions. This indicates the current level of model uncertainty of how to calculate the details of a cascade of interacting hadrons. Given this range of model systematics it is premature to claim the existence of in-medium masses using a model comparison of kaon yields [16, 18].

The situation can be improved by constraining the models to reproduce the high precision $\mathrm{p}+\mathrm{p} \rightarrow \mathrm{K}^{+}$data from COSY [19], and the semi-inclusive $\mathrm{p}+\mathrm{A}$ data at AGS energies that is becoming available. As an example the yield of $\mathrm{K}_{s}^{0}$ in $\mathrm{p}+\mathrm{Au}$ at $18 \mathrm{GeV} / \mathrm{c}$ from $\mathrm{E} 910$ [20] is shown in Figure 7. The yield increases rapidly with the number of slow particles associated with the reaction, consistent with the hypothesis that as the proton suffers multiple collisions, 
it increases both strangeness and the overall yield of particles. Successful transport models should be able to reproduce the wide range of $\mathrm{p}+\mathrm{p}, \mathrm{p}+\mathrm{A}$ reactions as an entry step towards the harder problems of $\mathrm{A}+\mathrm{A}$ reactions.

\section{KAON SPECTRA}

Insight into the dynamics of particle production and propagation can be obtained by examining the spectra of kaons. In the top panels of Figure 8 , dN/dy distributions for $\mathrm{K}^{+}$and $\mathrm{K}^{-}$are shown for a mid-central event class of $\mathrm{Au}+\mathrm{Au}$ reactions at $11.6 \mathrm{AGeV} / \mathrm{c}$ [9]. These have been extracted from exponential fits to invariant spectra as a function of transverse mass. Also extracted from each transverse spectrum is the inverse slope, T. The rapidity dependence of the inverse slope parameter is shown in the lower panels of Figure 8. The $\mathrm{dN} / \mathrm{dy}$ distribution for $\mathrm{K}^{-}$is narrower than for $\mathrm{K}^{+}$, and the $\mathrm{K}^{+}$inverse slopes tend to be slightly larger than the inverse slope for $\mathrm{K}^{-}$. Both observations are consistent with there being less phase space available for $\mathrm{K}^{-}$due to the higher energy threshold for $\mathrm{K}^{-} \mathrm{K}^{+}$pair production compared to $\mathrm{K}^{+} \Lambda$ associated production.

In contrast, the transport model RQMD predicts that the $\mathrm{K}^{-}$inverse slopes are larger than those for $\mathrm{K}^{+}$. This can be seen in Figure 9 which plots the difference of slopes, $\mathrm{T}_{K+}-\mathrm{T}_{K-}$. The incorrect ordering of slopes in RQMD could be caused by a too strong absorption of low $\mathrm{m}_{t} \mathrm{~K}^{-}$effectively increasing the $\mathrm{K}^{-}$inverse slope. This could merely be a problem with the parameterization or alternatively it could indicate the need to include either multi-body collisions [3] or an attractive in-medium potential to the transport model. This would effectively soften the $\mathrm{K}^{-}$spectra as has been observed in the in-medium HSD calculations for $\mathrm{Ni}+\mathrm{Ni}$ reactions at $1.8 \mathrm{AGeV}[18]$.

In addition to the softening of spectra, a rather striking low- $\mathrm{p}_{t}$ rise has been predicted in the $\mathrm{K}^{-}$spectra due to the attractive mean-field [16]. In preliminary E864 data [21] at the AGS there is no observation of such a rise even at values of $\mathrm{m}_{t}-\mathrm{m}_{0}=50 \mathrm{MeV}$. At GSI the predicted rise occurs just beyond the current experimental acceptance [16]. 
One sensitive probe of the possible existence of in-medium kaon properties is collective flow. Figure 10 shows the measure $\mathrm{K}^{+}$squeezeout [22], or preferential emission at azimuthal angles $90^{\circ}$ and $270^{\circ}$ from the event reaction plane, in $\mathrm{Au}+\mathrm{Au}$ reactions at $1 \mathrm{AGeV}$. The RBUU calculations that include an in-medium repulsive kaon potential better reproduce the measured azimuthal anisotropy.

\section{STRANGENESS AS QGP SIGNATURE}

The kaon production rate from a QGP has been predicted to be larger than the hadronic production rate, leading to the suggestion that strangeness enhancement is a plausible QGP signature [23]. As the beam energy is increased from 1 to $10 \mathrm{AGeV}$, the density of the participating matter should increase [24]. A small but increasing volume of QGP might be observable as an additional increase in kaon production beyond the hadronic enhancement from multiple collisions. This would not necessarily be a sharp onset with beam energy, but rather a change of evolution in strange yields.

The mid-rapidity yields of $\pi^{+}$and $\mathrm{K}^{+}$as a function of the initial available energy $\sqrt{s}$ are shown in the upper panels of Figure 11. Both pion and kaon yields increase steadily and smoothly with beam energy. There is no statistically significant indication of any sudden increase, or change in evolution of the particle yield with increasing $\sqrt{s}$.

In the lower panels of Figure 11, the $\left\langle m_{t}>\right.$ of the transverse spectra minus the rest mass for $\pi^{+}$and $\mathrm{K}^{+}$are plotted versus $\sqrt{s}$. The $\left\langle m_{t}>\right.$ is calculated from each fit to the transverse spectrum and hence includes an extrapolation to low $\mathrm{m}_{t}$. Compared to the increase of particle production, the $\left\langle m_{t}>\right.$ increases slowly with beam energy. The extra available energy mainly goes into more particle production rather than increased transverse energy.

The increase in kaon mid-rapidity yield with beam energy in Figure 11 is more rapid than the increase of pion yield. This is emphasized in Figure 12, where the $K^{+} / \pi^{+}$ratio of $\mathrm{dN} / \mathrm{dy}$ is plotted versus $\sqrt{s}$. This ratio increases steadily from near $3 \%$ at $2 \mathrm{~A} \mathrm{GeV}$ to $19 \%$ 
at $10.7 \mathrm{~A} \mathrm{GeV}$. The mid-rapidity $\mathrm{K}^{+} / \pi^{+}$ratio from central $\mathrm{Au}+\mathrm{Au}$ reactions at $1 \mathrm{~A} \mathrm{GeV}$ from the KaoS collaboration $\left[13\right.$ is $\mathrm{K}^{+} / \pi^{+}=(3 \pm 1) \times 10^{-3}$. This is one order of magnitude below the $\mathrm{K}^{+} / \pi^{+}$ratio at $2 \mathrm{~A} \mathrm{GeV}$. Also shown in this figure is the $K^{+} / \pi^{+}$ratio measured in central $\mathrm{Pb}+\mathrm{Pb}$ reactions at $\mathrm{CERN}$ [25]. This ratio is comparable, if somewhat lower, than the AGS result.

The double ratio, the measured $\mathrm{Au}+\mathrm{Au} \mathrm{K}^{+} / \pi^{+}$ratio divided by the $\mathrm{p}+\mathrm{p} \mathrm{K}^{+} / \pi^{+}$ratio is shown in Figure 13. This double ratio is greater than one, demonstrating that $\mathrm{K}^{+} / \pi^{+}$ is enhanced in $\mathrm{Au}+\mathrm{Au}$ reactions relative to $\mathrm{p}+\mathrm{p}$ collisions. This enhancement steadily decreases and is low at SPS beam energies, possibly because secondary collisions decrease in relative importance for kaon production compared to initial collisions as the beam energy is increased. A steady decrease in enhancement coupled with the smooth increase in the $\mathrm{K} / \pi$ ratio from $\mathrm{p}+\mathrm{p}$ reactions naturally produces a maximum in the $\mathrm{K} / \pi$ ratio in $\mathrm{A}+\mathrm{A}$ reactions.

\section{UPCOMING RESULTS}

Many new measurements on strangeness have recently been made. At GSI the KaoS collaboration is analysing proton and carbon-induced reactions to provide a baseline for kaon production via multiple secondary collisions. Detailed $\mathrm{K}^{+}$and $\mathrm{K}^{-}$spectra at $4 \mathrm{AGeV}$ from E866@AGS will provide more information on possible in-medium effects. Complementing the kaon data is the measurement of the $\Lambda$ excitation function by E895@AGS. Multi-strange production from $\mathrm{Au}+\mathrm{Au}(\mathrm{E} 896)$ and $\mathrm{p}+\mathrm{A}$ reactions (E910) at the AGS will be soon available to compare to the results at SPS. In addition E917@AGS is analysing the centrality dependence of anti-lambda as well as the mass, width of the decay $\phi \rightarrow \mathrm{K}^{+} \mathrm{K}^{-}$.

\section{CONCLUSIONS}

The availability of heavy-ion data at beam energies of 2, 4, 6, $8 \mathrm{AGeV}$ has bridged the gap between BEVALAC/SIS and AGS accelerators. This provides the opportunity to systematically study the properties of strongly-interacting matter as a function of the 
density. Two intriguing possibilities are the whether hadrons change their properties in a dense medium, or whether the baryon-rich quark-gluon plasma can be found.

The observation of $\mathrm{K}^{+}$squeezeout in $\mathrm{Au}+\mathrm{Au}$ reactions at $1 \mathrm{AGeV}$ is consistent with the presence of a repulsive kaon potential. However transport models that include such effects underpredict the yield of $\mathrm{K}^{+}$. In-medium effects are also predicted to increase the yield of $\mathrm{K}^{-}$, potentially changing the $\mathrm{K}^{-} / \mathrm{K}^{+}$ratio as a function of centrality and causing a sharp low $\mathrm{p}_{t}$ rise in the $\mathrm{K}^{-}$spectra. Neither effect is observed.

Taking the above data together as a whole, there is no consistent evidence for kaon inmedium properties. In addition using model comparisons to extract this physics is difficult. The factor of two discrepancy between different models with nominally the same physics input indicates the current level of uncertainty in how to calculate a cascade of interacting hadrons. Given this range of model systematics it is premature to claim the existence of in-medium masses using a model comparison of kaon yields.

At densities four to five times normal nuclear matter densities multi-body (as opposed to two-body) collisions could dominate the dynamics. This topic needs more attention, in particular how to distinguish multi-body collisions from a succession of two-body interactions.

The measured particle yields and the $\mathrm{K}^{+} / \pi^{+}$ratio smoothly evolve with beam energy between 1 and $10 \mathrm{AGeV}$. There is no indication of a change in behavior that could indicate the onset of a baryon-rich QGP. By establishing an energy dependence of particle production, the data provide a stronger link between AGS and SPS energies. Interpolating between the newly available excitation function and the full SPS energy leads to empirical predictions for quantities such as the $\mathrm{K} / \pi$ ratio at energies of 30-40 $\mathrm{AGeV}$, the planned low-energy run at the SPS. The extension of the excitation function from SIS $\rightarrow$ AGS $\rightarrow$ SPS will provide a new opportunity to examine how strongly interacting matter evolves with density and whether there is any indication of the onset of the quark-gluon plasma. 


\section{REFERENCES}

[1] Proceedings of Quark Matter '96, Nucl. Phys. A610, (1996).

[2] G.E. Brown et al., Nucl. Phys. 567, 937 (1994).

[3] C.A. Ogilvie, to be published, Phys. Lett. B

[4] M. Alford et al., Phys. Lett B 422247 (1998), K. Rajagopal hep-ph/9808348.

[5] W. Weise, Nucl. Phys. A610, 35c (1996)

[6] B.-A. Li and C.M. Ko, Phys. Rev. C 54, 3283 (1996).

[7] W. Ehehalt and W. Cassing, Nucl. Phys. A602, 449 (1996).

[8] X.S. Fang et al., Phys. Rev. C 47, 1678 (1993).

[9] , L. Ahle et al, E866 Collaboration, Phys Rev C, Nov 1998.

[10] H. Sorge, H. Stöcker and W. Greiner , Ann. Phys. (NY) 192, 266 (1989).

[11] Y. Pang, T.J. Schlagel, and S.H. Kahana, Phys. Rev. Lett. 68, 2743 (1992).

[12] B-A. Li and C.M. Ko, Phys. Rev. C 52, 2037 (1995) and B-A. Li private communication (1998).

[13] D. Miskowiec et al., KaoS Collaboration, Phys. Rev. Lett, 72,3651 (1994), and P. Senger, private communication (1998).

[14] E. Anderson et al., WA97 Collaboration, to be published Phys. Lett. B, and CERN$\mathrm{EP} / 98-64$.

[15] R. Barth et al, KaoS Collaboration, Phys. Rev. Lett. 78, 4007 (1997)

[16] C.M. Ko, G.Q. Li, J. Phys G22, 1673, (1996), G.Q. Li, nucl-th/9702023, and G.Q. Li, nucl-th/980413.

[17] S. Bass et al., Prog. Part. Nucl. Phys. 41, 225 (1998). 
[18] J. Geiss et al, nucl-th/9805012.

[19] J.T. Balewski et al., Phys. Lett. B 388, 859 (1996) and D.Grzonka Nucl. Phys. A631, 262c (1998).

[20] B.Cole E910 Collaboration, Quark Matter 1997.

[21] N. George, E864 Collaboration, private communication (1998).

[22] Y. Shin et al., KaoS Collaboration, nucl-ex/9807003

[23] J. Rafelski and B. Müller, Phys. Rev. Lett. 48, 1066 (1982).

[24] There is currently no proof that the baryon-density of participant matter increases monotonically between 1 and $10 \mathrm{AGeV}$. At some beam energy it is expected that a maximum density is reached.

[25] P. Seyboth, NA49, private communication (1998). 


\section{FIGURES}

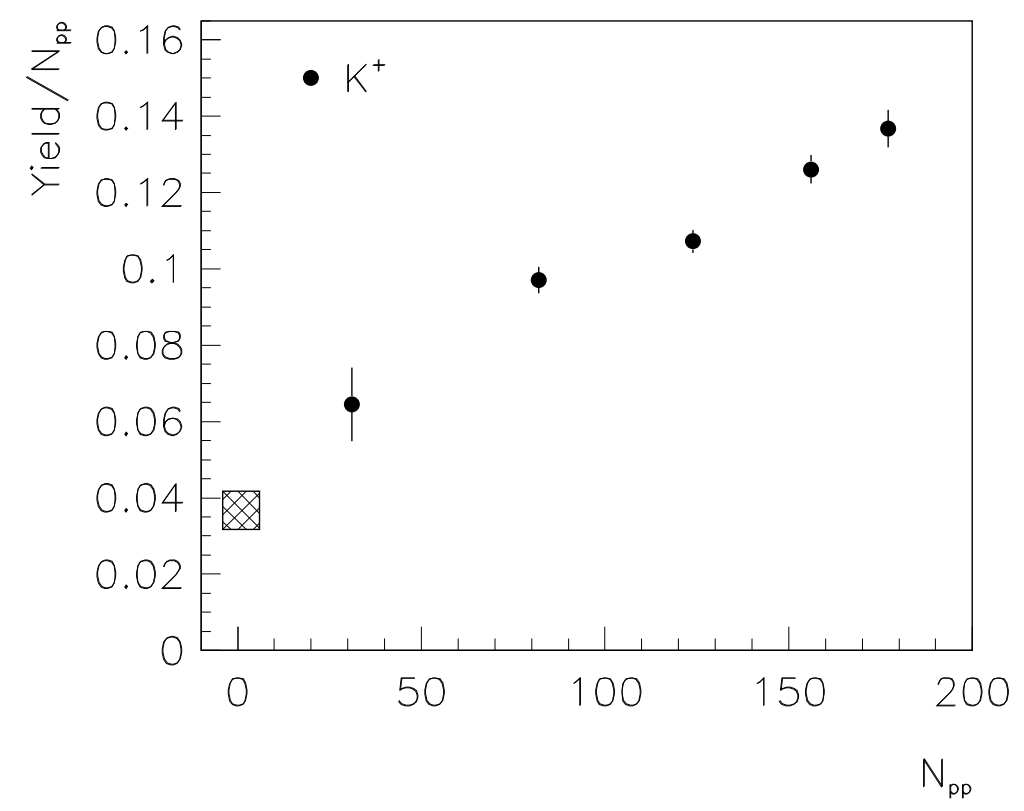

FIG. 1. The total yields of $\mathrm{K}^{+}$per projectile participant versus the number of projectile participants, $\mathrm{N}_{p p}$, in $\mathrm{Au}+\mathrm{Au}$ reactions at $11.6 \mathrm{~A} \mathrm{GeV} / \mathrm{c}$ measured by E866 [9]. The box on the left is an estimate of the kaon yield from the initial $\mathrm{N}+\mathrm{N}$ collisions. 


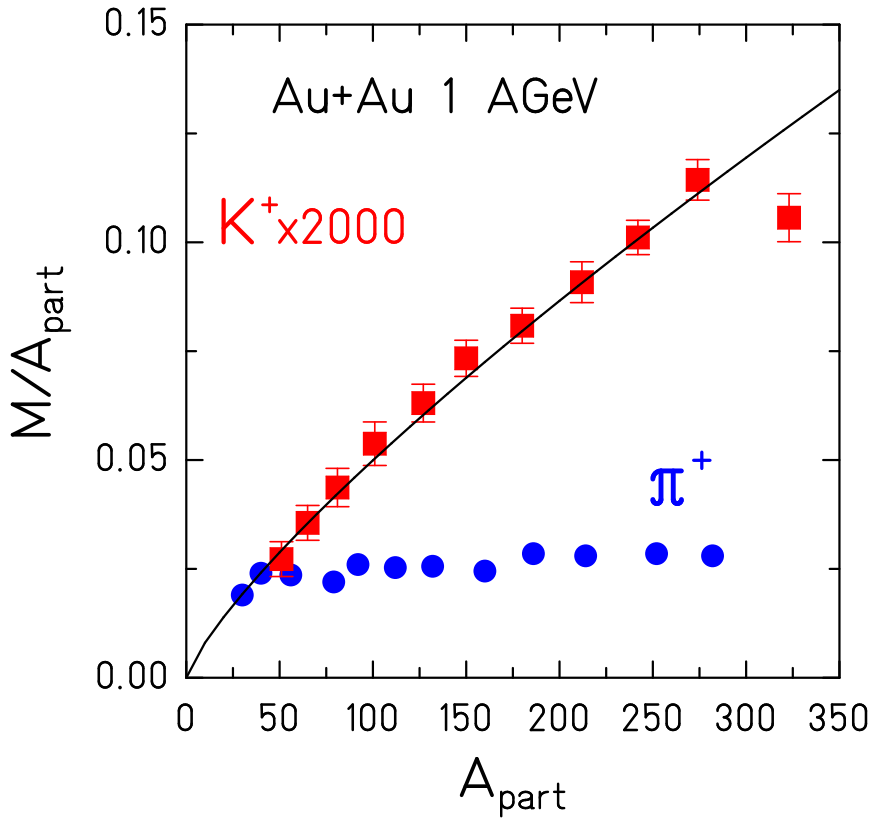

FIG. 2. The total yields of $\mathrm{K}^{+}$and $\pi^{+}$per participant versus the number of participants, $\mathrm{A}_{\text {part }}$, in $\mathrm{Au}+\mathrm{Au}$ reactions at $1 \mathrm{~A} \mathrm{GeV}$ measured by KaoS [13]. 


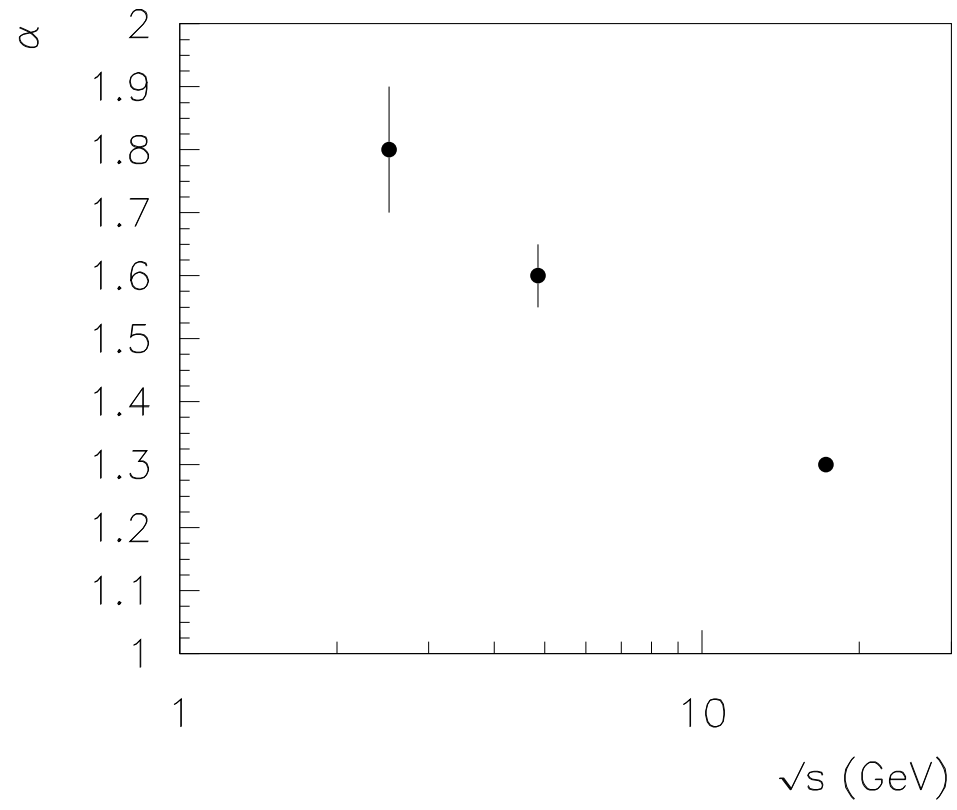

FIG. 3. The beam energy dependence of the power-law exponent $\alpha$ (equation 1) that describes the non-linear increase of strangeness production with centrality. 


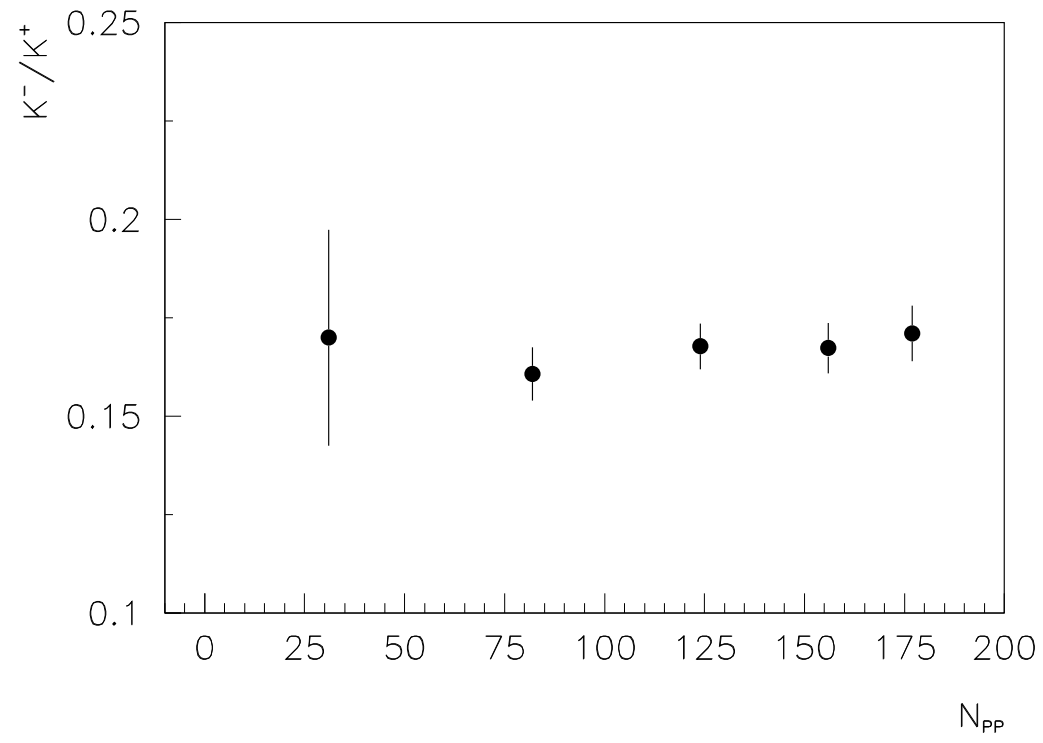

FIG. 4. The $\mathrm{K}^{-} / \mathrm{K}^{+}$ratio of total yields versus the number of projectile participants, $\mathrm{N}_{p p}$, in $\mathrm{Au}+\mathrm{Au}$ reactions at $11.6 \mathrm{~A} \mathrm{GeV} / \mathrm{c}$ measured by E866 [9]. 


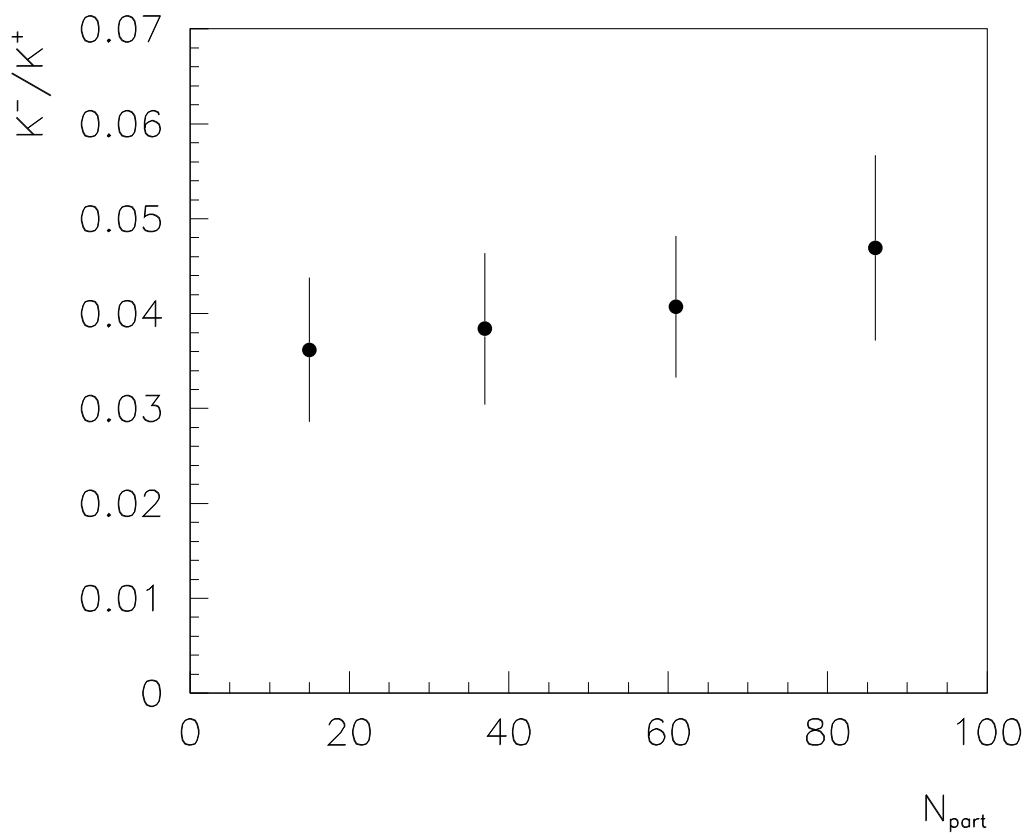

FIG. 5. The $\mathrm{K}^{-} / \mathrm{K}^{+}$ratio of total yields versus the number of participants in $\mathrm{Ni}+\mathrm{Ni}$ reactions at $1.8 \mathrm{~A} \mathrm{GeV}$ measured by KaoS [15]. 


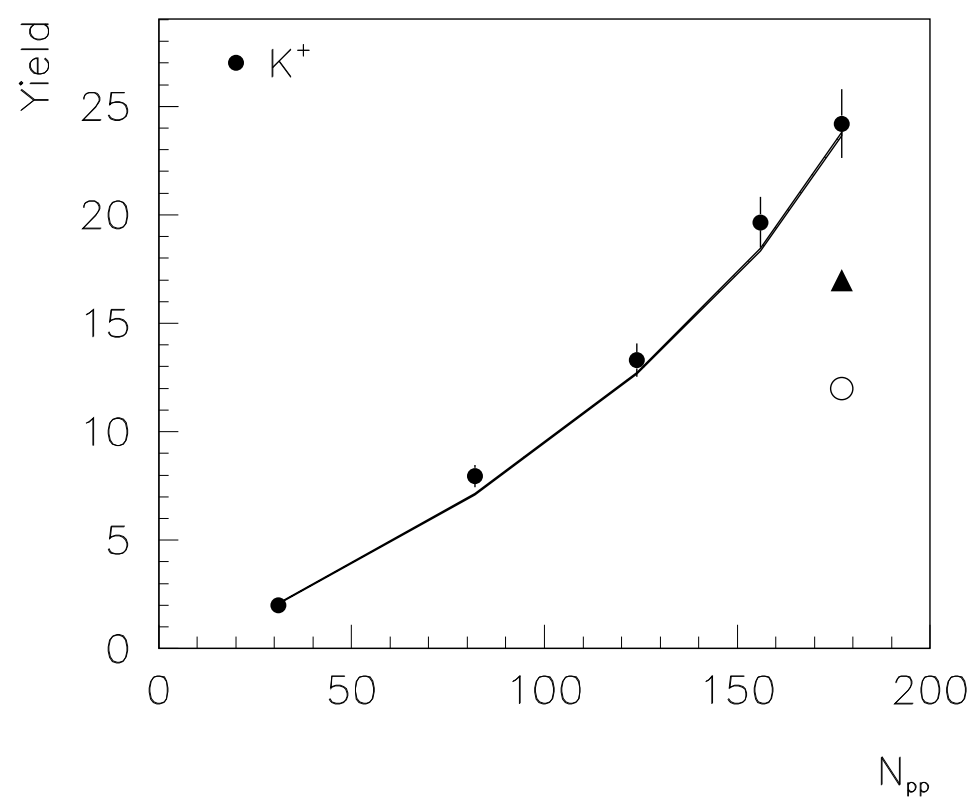

FIG. 6. The measured $\mathrm{K}^{+}$yield in $\mathrm{Au}+\mathrm{Au}$ reactions at $11.6 \mathrm{~A} \mathrm{GeV} / \mathrm{c}$ versus the number of projectile participants [9]. The data are compared to the hadronic transport model RQMD (v2.3) [10] (solid line) and the HSD [7] (open circle) and ART [6] (filled triangle) models run with normal hadronic masses. 


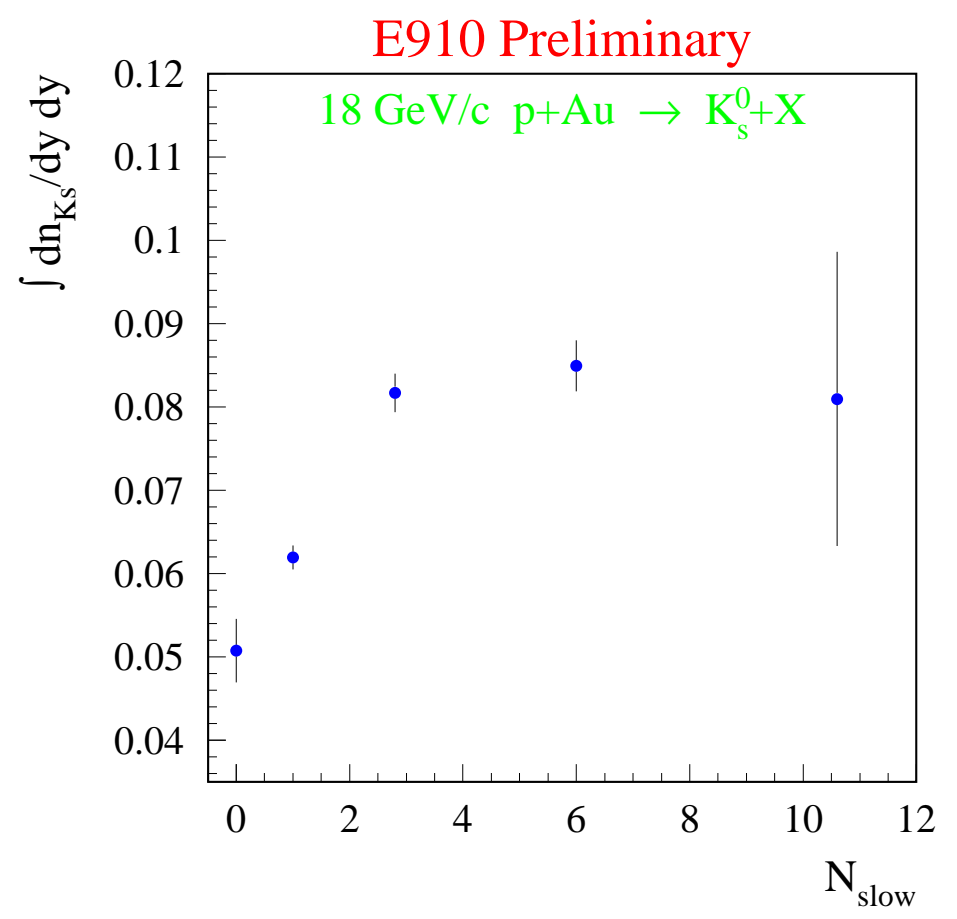

Analysis by X. Yang, Columbia Univ.

FIG. 7. The preliminary integrated $\mathrm{K}_{s}^{0}$ yield in $\mathrm{p}+\mathrm{Au}$ reactions at $18 \mathrm{GeV} / \mathrm{c}$ versus the number of slow particles emitted in the event as measured by E910 [20]. 

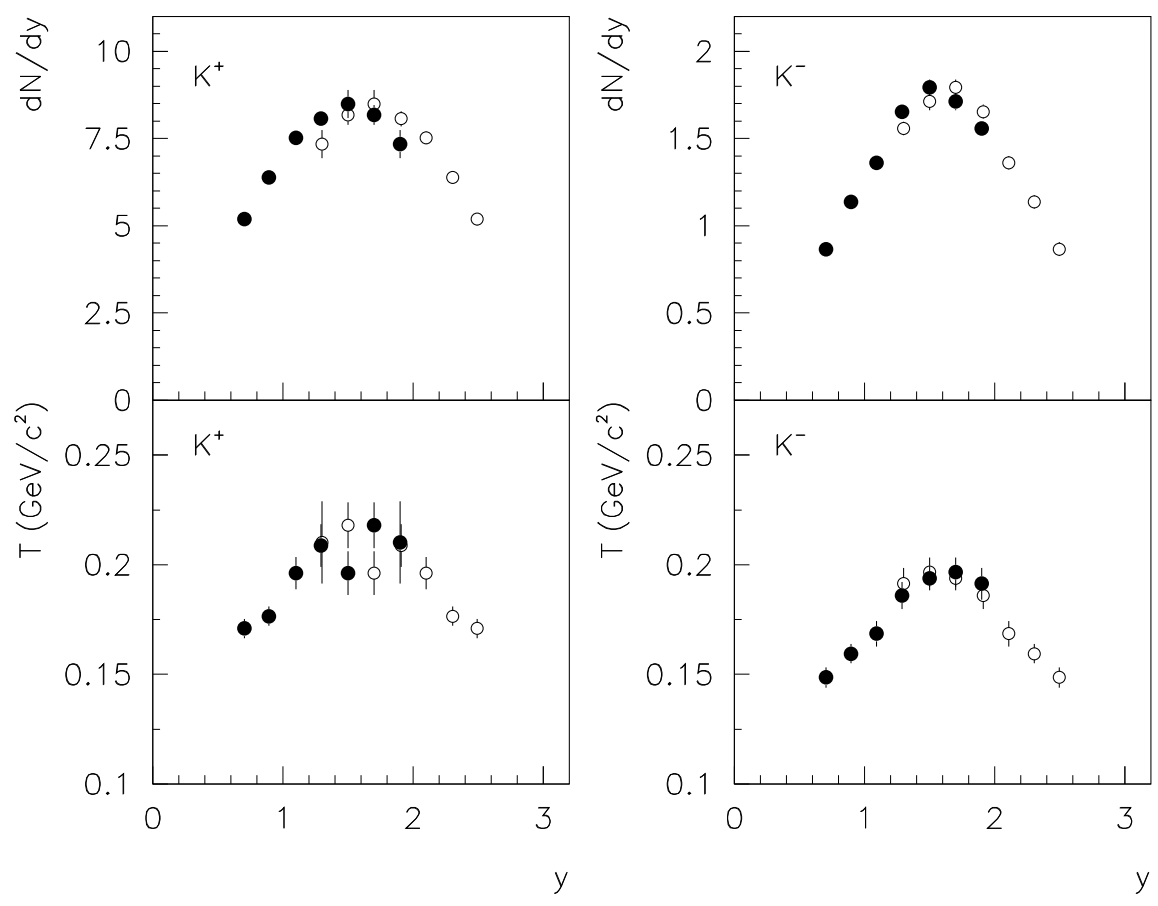

FIG. 8. The $\mathrm{dN} / \mathrm{dy}$ and inverse slope distributions as a function of rapidity for $\mathrm{K}^{+}$and $\mathrm{K}^{-}$ emitted from mid-central $\mathrm{Au}+\mathrm{Au}$ reactions at $11.6 \mathrm{~A} \mathrm{GeV} / \mathrm{c}$ [9]. The hollow circles are the data points reflected about mid-rapidity $(\mathrm{y}=1.6)$. 


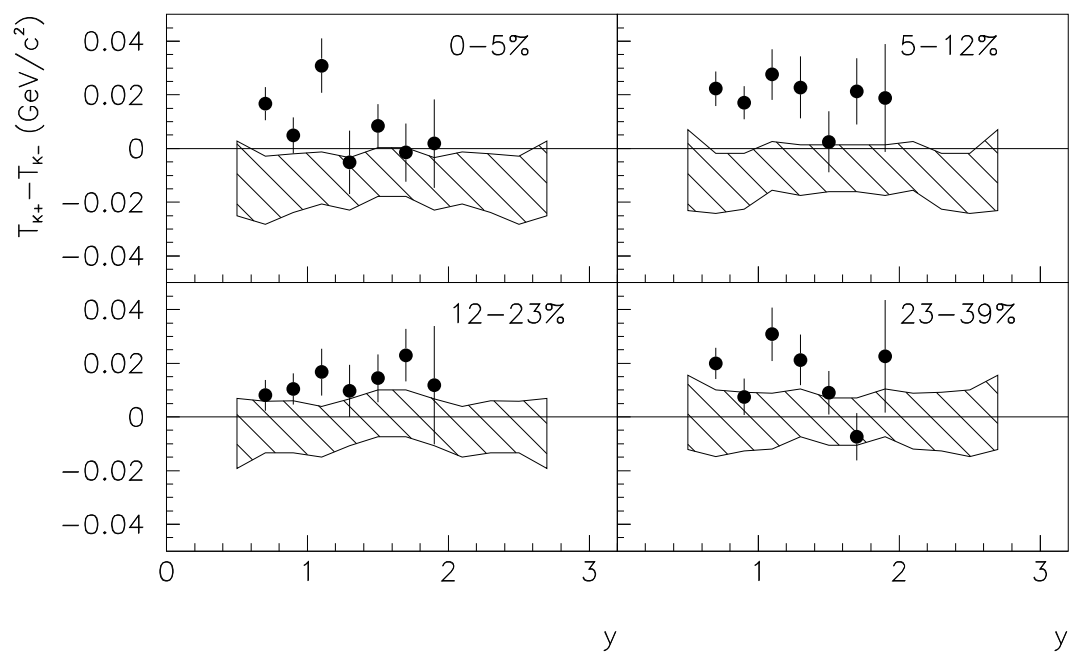

FIG. 9. The difference in inverse slope parameter $\mathrm{T}_{K^{+}}-\mathrm{T}_{\mathrm{K}^{-}}$versus $\mathrm{y}$ for the different centrality classes in $\mathrm{Au}+\mathrm{Au}$ reactions at $11.6 \mathrm{~A} \mathrm{GeV} / \mathrm{c}$. The centrality classes are categorized by the $\%$ of total reaction cross-section $(6.8 \mathrm{~b})[9]$. The data are compared to the hadronic transport model RQMD (v2.3) [10] . The dashed band is centered at the model prediction and has total width of $\pm 1 \sigma$. 


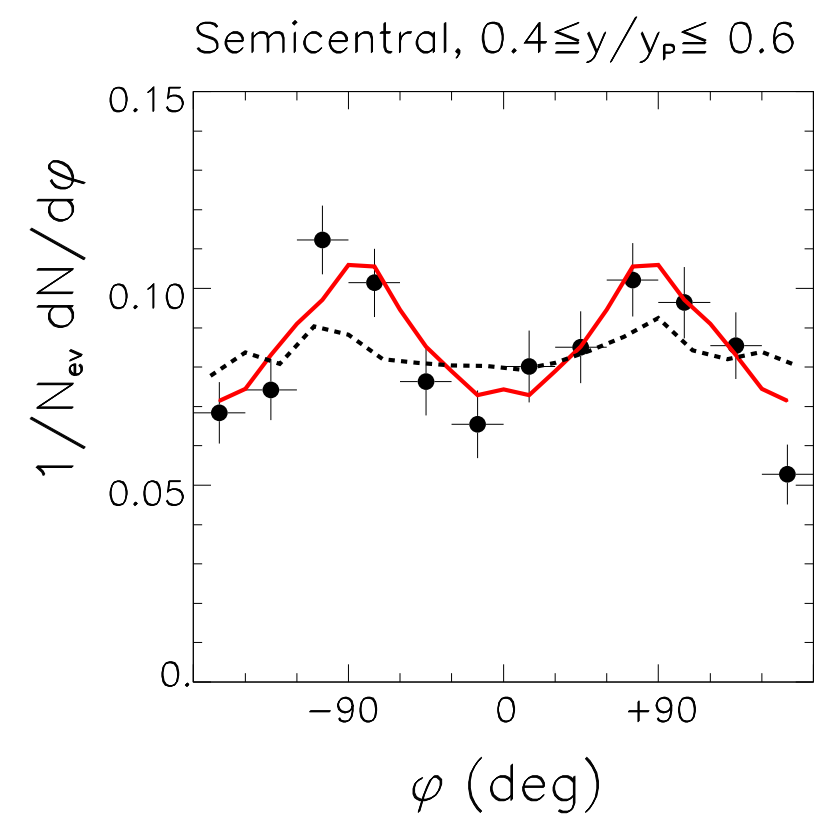

FIG. 10. The azimuthal distribution of $\mathrm{K}^{+}$in semi-central $\mathrm{Au}+\mathrm{Au}$ collisions at $1 \mathrm{AGeV}$ as measured by KaoS [22]. The lines represent RBUU calculations for an impact parameter of $b=7 \mathrm{fm}$ without (dashed line) and with an in-medium KN potential (solid line). 

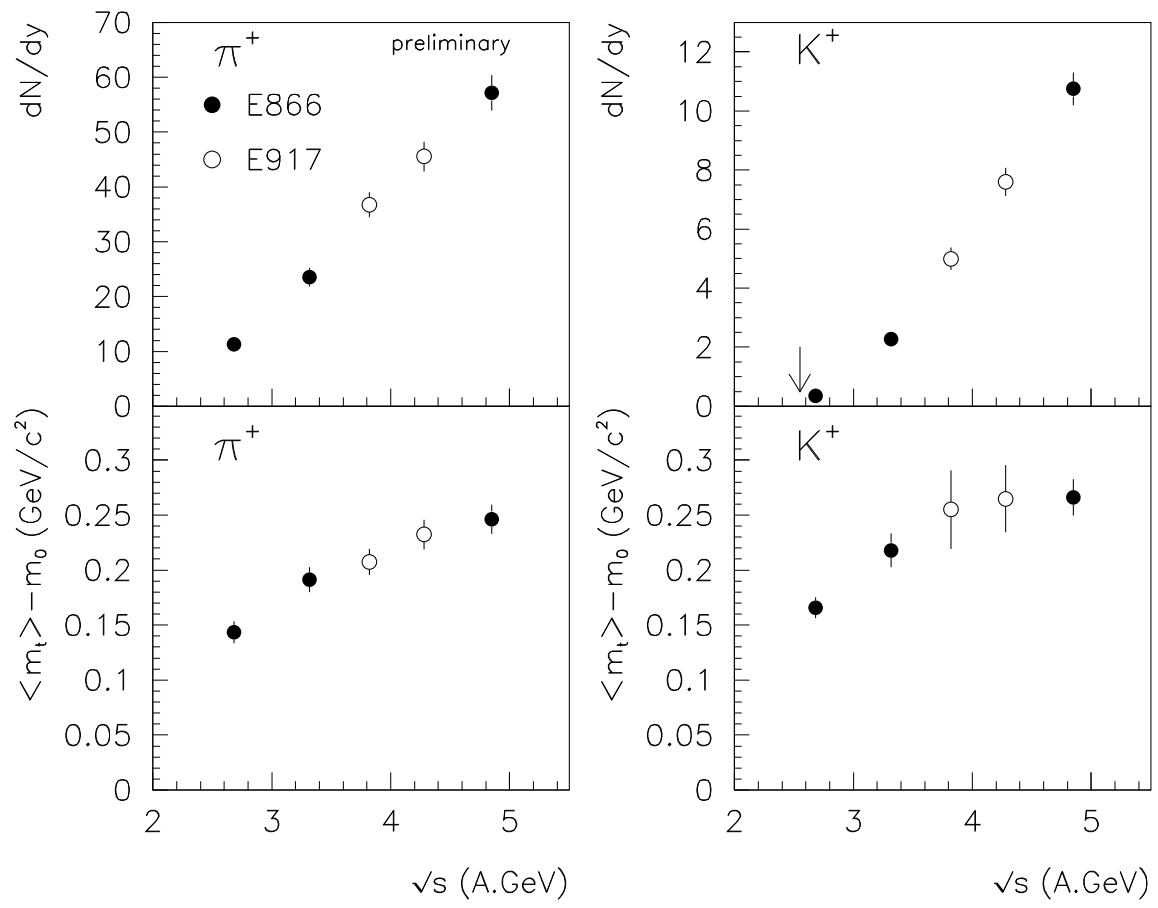

FIG. 11. The yield of $\pi^{+}$and $\mathrm{K}^{+}$at mid-rapidity (top-panels) for central $\mathrm{Au}+\mathrm{Au}$ reactions as a function of the initial available beam energy. The lower panels show the mean $\mathrm{m}_{t}$ minus the rest mass for $\pi^{+}$and $\mathrm{K}^{+}$at the same rapidity. 


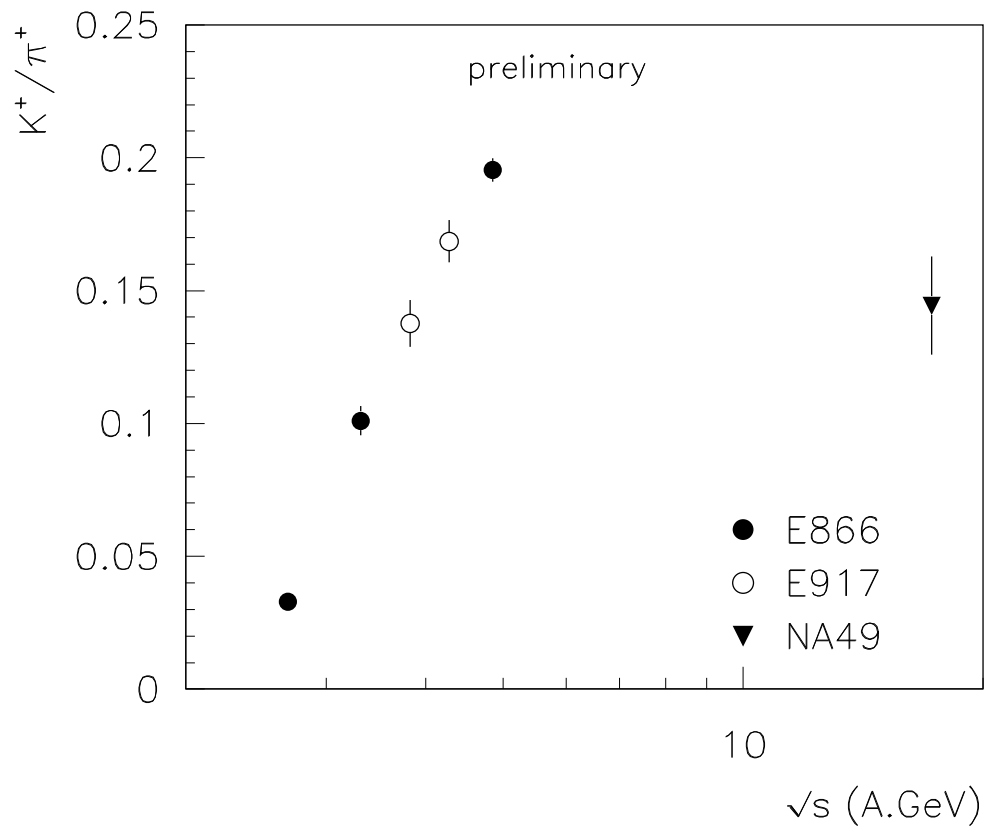

FIG. 12. The ratio $\mathrm{K}^{+} / \pi^{+}$at mid-rapidity in central $\mathrm{Au}+\mathrm{Au}$ reactions as a function of the initial available energy. 


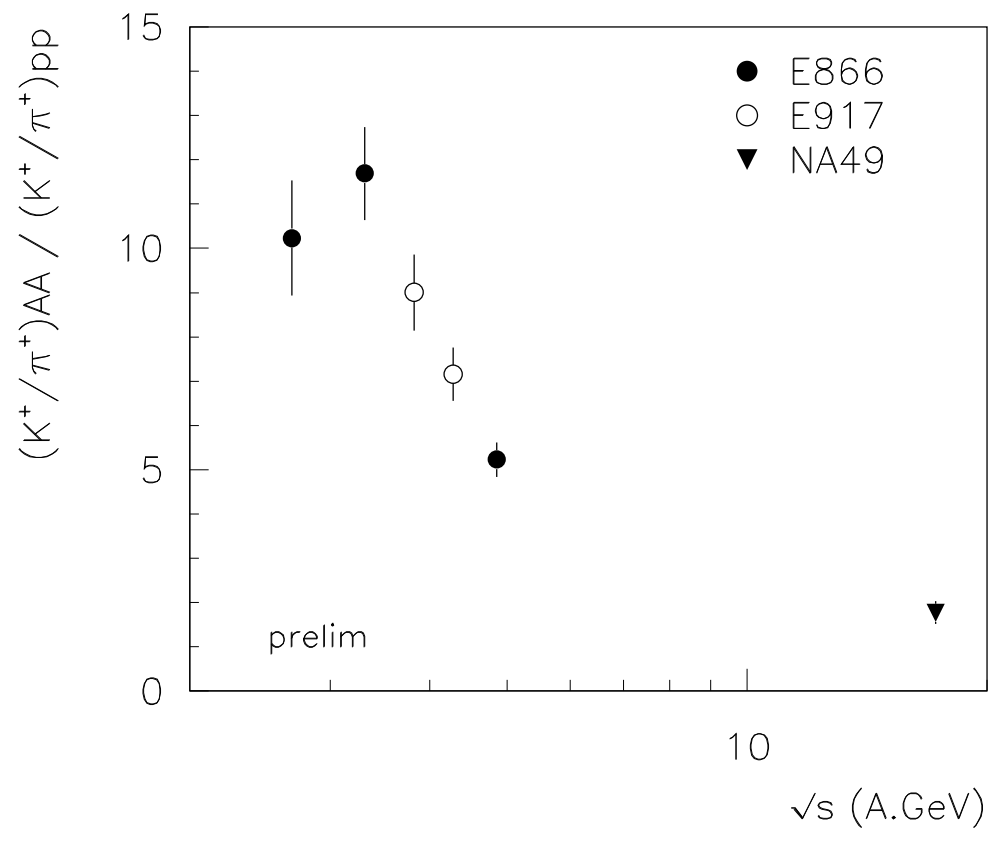

FIG. 13. The double ratio $\mathrm{K}^{+} / \pi^{+}$at mid-rapidity from central $\mathrm{Au}+\mathrm{Au}$ reactions divided by $\mathrm{K}^{+} / \pi^{+}$of total yields from $\mathrm{p}+\mathrm{p}$ reactions as a function of the initial available energy. 